complicated initial febrile seizures and ES are also at greater risk of developing epilepsy

\title{
EPILEPSY AND COGNITIVE PERFORMANCE
}

Changes in left temporal interictal epileptiform activity during and after the performance of cognitive tasks are reported in an 18-year-old male with intractable complex partial seizures evaluated at the Radcliffe Infirmary, Oxford, UK. Epileptiform discharges were suppressed or enhanced depending on the nature and timing of the tasks. A posterior temporal spike focus occurred only during rest periods that followed verbal tasks. The mean left mid-to-anterior temporal spike count was halved during verbal tasks compared to the count during visuo-spatial tasks. A relatively low rate of right-sided temporal discharges was unchanged during or after the performance of tasks. (Boniface SJ et al. Changes in focal interictal epileptiform activity during and after the performance of verbal and visuospatial tasks in a patient with intractable partial seizures. I Neurol Neurosurg Psychiatry Feb 1994; $57: 227-228$ ). (Respond: Dr SJ Boniface, Dept of Clinical Neurophysiology, Radcliffe Infirmary, Woodstock Road, Oxford OX2 6HE, UK).

COMMENT. Activation or suppression of EEG focal spike discharges in relation to psychological testing may have a role in the medical treatment of certain children with reading and other learning disabilities as well as the assessment of patients before and after surgery for epilepsy.

\section{ANTIEPILEPTIC DRUGS}

\section{PHENYTOIN AND COGNITIVE-MOTOR PERFORMANCE}

Cognitive-motor function in 51 children with seizures well controlled with phenytoin (PHT) monotherapy was assessed in relation to drug concentration, seizure type, and time of medication at the Departments of Psychiatry and Pharmacology, Auckland University School of Medicine, Australia. Age ranged from 4 to 14 years, and performance was significantly better in older patients. Diagnosis (partial vs generalized epilepsy), PHT concentration levels, and change from trough to peak concentration days had little effect. Fluctuations in PHT as great as $50 \%$ had no or minimal effects on performance of tests in low therapeutic doses. (Aman MG, Werry JS et al. Effects of phenytoin on cognitive-motor performance in children as a function of drug concentration, seizure type, and time of medication. Epilepsia Jan/Feb 1994;35:172-180). (Reprints: Dr MG Aman, Nisonger Center, Ohio State University, 1581 Dodd Dr, Columbus, OH 43210).

COMMENT. Maintenance phenytoin monotherapy, at relatively low therapeutic levels, had negligible or no effects on cognitive motor function in a group of children with well controlled seizures.

Performance swings resulting from drug absorption and elimination were absent or minimal in this carefully monitored study. The importance of frequent determinations of phenytoin levels during evaluations of neuropsychological function in children is evident from the following report. 